\title{
The Research of the technology on computer adaptive testing
}

\author{
Chunxue $\mathrm{Xu}^{1, \text { a }}$, Jia Zhu ${ }^{1, a}$ \\ ${ }^{1}$ School of Computer Science,Baicheng Normal University \\ a 8zj26@163.com
}

Key words: computer adaptive testing; key technology; research

\begin{abstract}
: with the development of science and technology, computer technology has made great progress in various fields; it has been very widely used. In the selection of talents, our country has been using the traditional test system, such as the examination and entrance examination, etc. And the traditional test is mainly written in the main, there are a lot of drawbacks. The computer adaptive test system, very good to avoid the traditional written test in the wrong and the leakage problem In the application of computer technology,. The application of this system not only saves a lot of manpower and material resources, but also greatly improves the efficiency of testing, and makes a great contribution to the various types of testing in our country.
\end{abstract}

\section{Introduction}

With the continuous development of computer technology and progress, the computer adaptive testing system is also in the continuous improvement. In the development process, there are new problems are discovered and solved, so that the computer adaptive testing has been further optimized and improved. Therefore, in practical applications, computer adaptive testing has been more and more trust and more and more applications. This, in turn, promotes the further development of computer adaptive testing. In the future development and application of them, we need to study the key technologies of computer adaptive testing, to promote its continuous improvement, so as to better serve the talent selection ${ }^{[1]}$.

\section{Theoretical basis of computer adaptive testing}

Computer adaptive test theory is no longer based on classical test theory as a support, but in the modern measurement theory as a guide, is item response theory (IRT), item response theory is relatively obvious advantages, can provide a broader space for the future development of computer adaptive test.

The technology model is about item response. Theesponse theory that mathematical form reaction in subjects with potential material for the project a certain model, this probability model can describe the project characteristic curve of increasing function, and the probability of correct association project participants and between the various projects. Mathematical researchers have proposed a variety of IRT models such as logistic model, normal give model. According to a number of projects on the logistic model parameters can be divided into three main types. Are the single parameter logistic models, the double parameter logic this association model and three parameter logistic model? 
The technology characteristics are about item response. There are some limitations in the classical test theory. Since its development is mainly for the psychological and educational measurement work, there are still many problems in the technology. Among them, the project parameters mainly depend on the difficulty of the project will be different. In the test scores, there are some limitations, too much depends on the difficulty of the project application ${ }^{[2]}$, scores and answer the number of projects, the project is difficult, the score will be low. Because of the limitations of the test reliability, the test effect is worse. Item response theory is mainly based on the theory of potential material, the main principle is that the subjects face the test response by the psychological impact, reflects the relatively stable and can be used as dominate action response to the test, and the memory characteristics of the same reaction the.

\section{The advantages of computer adaptive testing}

The flexibility is high. When the computer is adaptive system about the test questions, this is according to the degree of master candidates and the actual level of knowledge, the automatic generation of test questions. This ensures that the control test was a good topic in the examinees' ability range, during the test; we can avoid the candidates due to too difficult problem, and have greater emotional impact, the pace of the test, thus affecting the overall strength of the level of play [1]. The test questions generated by the computer adaptive system is the production according to the actual level of examinees, so as to understand the comprehensive level of the corresponding test subjects better, make the test process with high flexibility.

Thetest accuracy is high. In the test questions in the traditional process, the degree of difficulty of the problem, it is difficult to effectively distinguish and control. The topic for the test subject can effectively control, so it is difficult to subject the degree of difficulty of accurate judgment, to the title of the test will be difficult to get good control ratio. And through this test subject to test the ability and level of the test, the accuracy of the results is also very limited. The computer adaptive system can be based on the actual level of the candidates ${ }^{[3]}$, the automatic test of the degree of difficulty of the subject to adjust. In this way, no matter how the results of the candidate, the test side can be a more accurate judgment of its ability and level, so that the accuracy of the test results to improve.

The cheating is avoided. The test questions are randomly generated by the system, In the computer adaptive test, and the test takers in the test, but also from the question bank randomly selected topics. In this way, it is able to effectively control and avoid the cheating behavior of the candidate in the test process. In addition, the probability of the same test items from the candidate is very low, very good suppression of the examination in whispers, pass notes cheating.

\section{Key technologies in computer adaptive testing}

The technology is about bank construction. It mainly includes the design and organization of the bank, and the determination and verification of the project development and project parameters In the construction of the question bank,. In the design and organization of item bank, it is mainly based on the structure of course catalog to construct and organize the test items. But this method has some limitations; someone should therefore, in the construction of domain ontology in the ontology technology, on the other hand, with the construction of the main body, to the corresponding point distribution of knowledge of project development. And the accuracy of the project parameters is one of the most important part, not only to accurately determine the initial value of all kinds of 
parameters, but also in the application of continuous maintenance.

The technology is about capability assessment. to the candidates, according to the actual result of the reaction, to the examinee's ability and level of evaluation, at the same time in the next test, after the project in response ,and to evaluate the new level and ability as a reference. Capacity assessment is mainly divided into two aspects; one is to set the initial ability of the candidate, on the other hand is in the test process, the ability of the corresponding test subjects to reevaluate. Therefore, it is not only to ensure the accuracy of the assessment, but also to ensure that the speed of the assessment in the ability evaluation technology.

The technology is about project selection. The amount of information is the most important factor, and it should be accurate calculation of the amount of information for each project In project selection technology ${ }^{[4]}$, while trying to improve the speed of calculation. For the uneven exposure impact on safety, test questions and other aspects of the use of the project, should take the exposure control, the discriminative item exposure rate is reduced, at the same time will use the low differentiation rate of project improvement. In practical application, should be on the project and the statistical indicators were also considered in the project selection process, the appropriate application of some content balance control technology, and provided some content, answer balance constraint processing technology. In addition, because the project selection method in practical application will be subject to certain restrictions, each of the choice of a project, it is to scan the entire question bank, the amount of computation increased. At the same time, if the multi user concurrent, server load will be even greater. Therefore, to use appropriate means to improve the speed of project selection.

The technology is about termination. It is shall be on the questions which test items used, candidates are tested in real time, and the corresponding test factors' ability to assess the precision of the comprehensive consideration and analysis In determining the test termination,. Therefore, in the test termination technology, it should be done to consider and analyze a variety of factors, so as to ensure the accuracy of the test results.

\section{Computer adaptive testing system}

The flow chart is test system on computer adaptive. Through the relevant theories, Theas a data source, a public course in Taiwan is used to implement the adaptive test system. Function mainly in order to achieve the fairness of the examination, so that modern teachers are no longer engaged in busy examination work, improve efficiency. The computer adaptive test flow chart is shown in figure 1. 


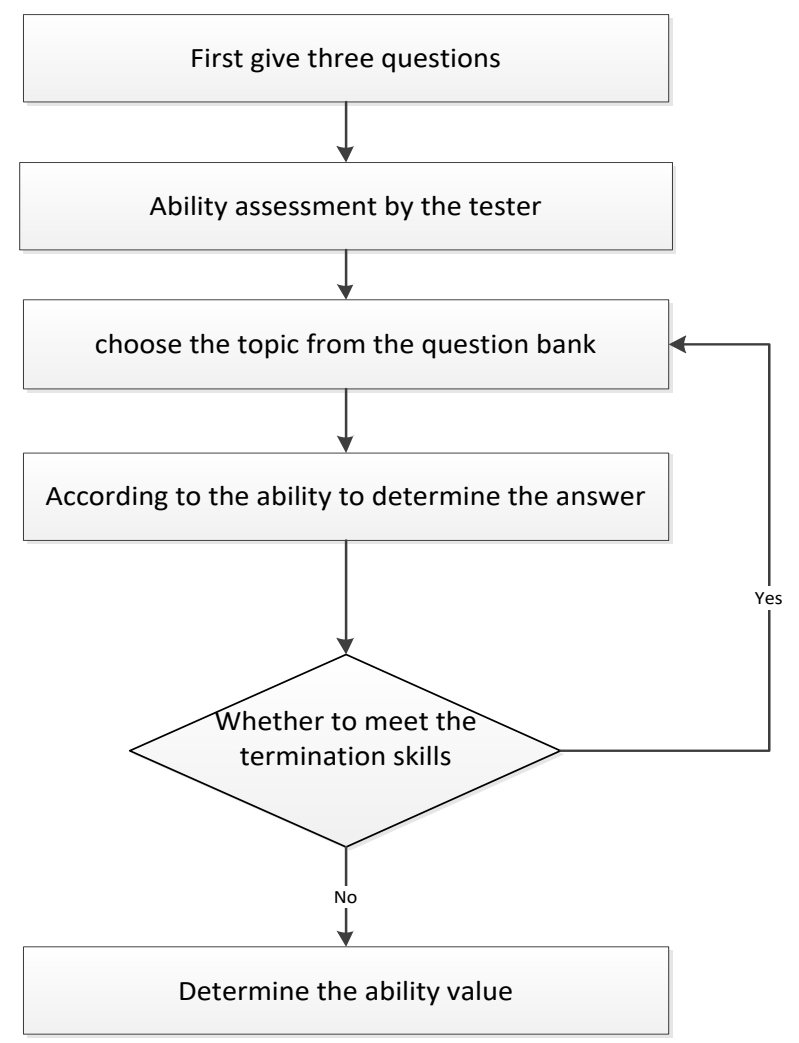

Fig.1 Flow chart of computer adaptive test system

The model is real. (1) Question bank management module of system module. Question bank is very important for the computer test, for adaptive experiments, is also a very important part of. First of all, it is to determine the IRT selection and subject parameters. From 2009 in Shandong to obtain a university capacity of a public class understanding, received a total of 1300 papers, can provide data support for the computer adaptive test. Through the analysis of these data, they found that with the three parameter logistic model fitting is relatively good, and the twenty item parameters are within the allowable range, so the need to put twenty items into a small bank. (2) Test management module. Computer adaptive test showed the same is also very important, how to present the topic, and termination of the development and management needs of examination management module, using Microsoft's.NET platform, calling the Oracle database to support background operation. We must first login interface and registered user interface for the design, mainly to let all the participants are able to see what questions they have done in the next time after landing, to focus on the wrong topic. In the exploration phase, three subjects were prepared for the subjects, so as to obtain the initial value of the initial test, to ensure that the accuracy of the measurement phase can be obtained with a short test length. In the termination of the examination system, the need to control its end in computerized adaptive test of a public class in the exam because of a limited number, there are some limitations, so it is necessary to estimate the standard deviation of the fixed capacity of fixed length of test method on the end of the test to take control.

\section{Conclusions}

In the current field of talent selection and testing, computer adaptive testing is one of the most ideal is also the most effective way to test a test. By using this kind of testing technology, it is able to 
carry on the comprehensive, comprehensive and accurate assessment of the level and ability of the subjects. In the process of design and application of computer adaptive test, to study and fully understand the entire key istechnology, so as to better improve, so that it can play a better effect.

\section{Reference}

[1]Jane small beads, Zhang Minqiang, Peng Chunmei. Test process and test technology of computer adaptive test[J]. education measurement and evaluation (THEORY EDITION), 2011,12:9-14.

[2] Wu Zhixin. Research on the application of computer adaptive testing technology based on [J]. XML MicrocomputerDevelopment, 2011, 2:137-139.

[3] Jane small beads, Zhang Minqiang, Peng Chunmei. Test process and test technology of computer adaptive test [J]. Journal of Chuzhou Vocational and Technical College, 2012,1:58-63.

[4] Liu Kunyang. Research and implementation of computer adaptive test technology [J]. computer CDSoftware and applications, 2014,2:97-98. 